\title{
Optimal Nonlinear Readout under Strong Non-Gaussian Noise
}

\author{
Akihisa ICHIKI* and Yukihiro TADOKORO \\ Toyota Central R\&D Labs., Inc., Nagakute, Aichi 480-1192, Japan
}

Received March 13, 2013; final version accepted May 30, 2013

\begin{abstract}
The optimal nonlinearity for readout a weak signal embedded in strong noise is investigated. In practical devices, a high signal-to-noise ratio (SNR) is preferred more than the Bayesian estimation, which generally requires complicated calculations. The optimal nonlinearity is determined by the noise statistics including its temporal correlations, and provides the highest SNR. According to the result, the existence of nonlinear devices that exhibit SNR higher than linear devices is shown under non-Gaussian noise. In contrast, linear devices exhibit the highest SNR in the presence of Gaussian noise. Using the resultant nonlinearity, the Cramér-Rao lower bound is realized by an easy linear estimator. Our derivation of the optimal characteristics of the filter gives the criterion to designed practical devices in the presence of noise.
\end{abstract}

KEYWORDS: nonlinear filter, non-Gaussian noise, colored noise, signal-to-noise ratio, linear estimator

\section{Introduction}

We focus on the extraction of a weak signal from noisy environment in this paper. The extraction of a weak signal is important in various fields, such as sensing, medical sciences, and telecommunications. The extraction of a weak signal that exhibits low signal-to-noise ratio (SNR) is a challenging problem, while conventional techniques sufficiently work for a strong signal compared to the background noise.

In many practical signal processing devices, high SNR is required, although a formal signal processing performance is given by Shannon entropy, which is completely different concept of the SNR. In order to obtain large Shannon entropy, statistical estimation techniques such as Bayesian estimation are used. However, such techniques require complicated calculations, and are not suitable for practical devices that perform their processing in real time and cost less. The SNR, which is an informal and intuitive indicator of signal processing performance, is thus preferred to use in practical device designing.

In order to design devices suitable for practical signal processing, we investigate the nonlinearity, which expresses the input-output characteristics of the devices, to maximize the SNR. The design of the optimal filter is a traditional target in filtering theory [1,2]. However, most of the traditional studies on the optimal filter is only fitting of several parameters [1]. Contrary, the optimal nonlinearity of the filter presented in this paper is truly optimal over all filters [3]. Such nonlinearity is expected to yield easy signal estimation, because SNR is an indicator of the signal processing performance in intuitive sense. Indeed, we will see that the optimal nonlinearity naturally leads to an easy estimator of which accuracy of signal estimation coincides with the Cramér-Rao lower bound $[4,5]$.

The remaining part of this paper is organized as follows: in the next section, we define the SNR in the presence of colored noise to prepare for the discussion on the maximization of SNR in general type of noise environment. In $\S 3$, we formulate the optimal nonlinearity in the presence of general types of noise. The result shows that linear system is the optimal in Gaussian noise environment, and that there exists suitable nonlinearity in the presence of non-Gaussian noise. As an example of non-Gaussian noise, mixed Laplace noise is used to compare the SNRs obtained from the optimal and linear filters. The relation between the optimal nonlinearity and the phenomenon called stochastic resonance (SR) is also discussed. The SR is the phenomenon where the SNR changes nonmonotonically with the monotonic change of the parameter characterizing the noise statistics. In addition, the optimal nonlinear filter for the noise generated from Markovian process is discussed. Such noise is important in practical situations, because various types of noises arising in practical engineering problems can be modeled by Markovian processes. In $\S 4$, we discuss on the relation of the optimal nonlinearity with signal estimation theory. We will see that the obtained nonlinearity yields the Cramér-Rao lower bound, which is the theoretical limit of signal estimation accuracy for unbiased estimators. In the last section, we briefly conclude this paper. 


\section{Definition of SNR under Colored Noise}

Before the discussion on maximization of SNR, we define SNR in the presence of colored noise. SNR is usually defined as the ratio of signal and noise powers at the signal frequency in Fourier power spectrum. This definition of SNR is well-defined when the noise is white. However, for colored noises, the definition of SNR using Fourier power spectrum does not describe the exact performance of signal extraction, because the noise component at the signal frequency can be estimated by the observation of noise component at different frequencies.

The signal extraction performance in principal is evaluated by the projection of the received signal. Consider the temporal sequence $\boldsymbol{S}+\boldsymbol{\xi}$, where the sequence of the weak signals $S=\left(S_{1}, \ldots, S_{N}\right)$ and the sequence of the background noises $\xi=\left(\xi_{1}, \ldots, \xi_{N}\right)$. The statistical properties of the noise sequence $\xi$ including its temporal correlation are arbitrary here. The signal extraction performance is well described by the ratio of signal and noise powers in Fourier spectrum in the presence of white noise. This is because the frequency gives a good basis to evaluate the magnitude of the signal component under white noise. Analogously to this fact, the definition of SNR in the presence of colored noise is given by the best basis to evaluate the magnitude of the signal. Therefore the definition of SNR in the presence of colored noise is given by the ratio of the magnitudes of the signal and noise components in the appropriate one-dimensional projection. Such a projection is formally expressed as

$$
X=\sum_{k=1}^{N} w_{k}\left(S_{k}+\xi_{k}\right),
$$

where the weights $\boldsymbol{w}=\left(w_{1}, \ldots, w_{N}\right)$ are determined so as to maximize the SNR $R_{X}$ for the projection $X$ :

$$
R_{X}=\frac{\left(\sum_{k=1}^{N} w_{k} S_{k}\right)^{2}}{\left\langle\left(\sum_{k=1}^{N} w_{k} \xi_{k}-\left\langle\sum_{l=1}^{N} w_{l} \xi_{l}\right\rangle\right)^{2}\right\rangle},
$$

where $\langle\cdots\rangle$ denotes the average with respect to the noise $\xi$. Note that $\sum_{k=1}^{N} w_{k} S_{k}$ and $\sum_{k=1}^{N} w_{k} \xi_{k}$ in the projection $X$ denote the signal and noise parts, respectively. The maximization condition for the SNR $R_{X}$, i.e., $\partial R_{X} / \partial w_{k}=0$ $(k=1, \ldots, N)$ yields the optimal weights $\boldsymbol{w}$ and the resultant SNR is given as

$$
R_{X}=\sum_{k=1}^{N} C_{k l}^{-1} S_{k} S_{l},
$$

where $C^{-1}$ is the inverse matrix of the covariant matrix of the noise $C_{k l}=\left\langle\xi_{k} \xi_{l}\right\rangle-\left\langle\xi_{k}\right\rangle\left\langle\xi_{l}\right\rangle$. Note that the definition of the SNR Eq. (2.3) is the natural extension of the SNR defined by the Fourier power spectrum in the presence of white noise. For a stationary white noise, the covariance matrix $C$ is given as $C_{k l}=\sigma^{2} \delta_{k l}$, where $\sigma^{2}$ is the variance of the noise and $\delta_{k l}$ denotes Kronecker's delta. Thus the SNR Eq. (2.3) for a stationary white noise is given as $R_{X}=\sum_{k=1}^{N} S_{k}^{2} / \sigma^{2}$, where $\sum_{k=1}^{N} S_{k}^{2} / N$ is the signal power and $\sigma^{2}$ is the noise power, and $R_{X}$ coincides with the SNR defined by Fourier power spectrum. We use the definition of SNR Eq. (2.3) throughout this paper.

\section{Optimal Nonlinearity}

\subsection{General derivation of optimal nonlinearity}

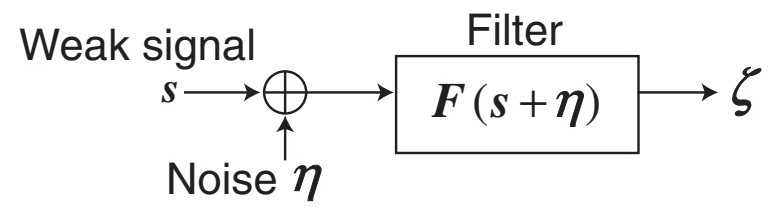

Fig. 1. Schematic image of nonlinear filtering system.

Consider the nonlinear filter that generates the output sequence $\zeta=\left(\zeta_{1}, \ldots, \zeta_{N}\right)$ from the input sequence $s+\eta$, where $s=\left(s_{1}, \ldots, s_{N}\right)$ and $\boldsymbol{\eta}=\left(\eta_{1}, \ldots, \eta_{N}\right)$ denote the sequences of the weak signal and noise, respectively. The schematic image of the system is shown in Fig. 1. Generally, each component of the output sequence $\zeta_{k}(k=1, \ldots, N)$ depends on the entire sequence of the input $s+\eta$ owing to the memory effect of the filter. Such a filter characteristics is expressed by its transfer function $\mathbf{F}=\left(F_{1}, \ldots, N\right)$ as

$$
\zeta_{k}=F_{k}(s+\eta)
$$

Note that the transfer function for each component of the output sequence $F_{k}$ differs for different $k$ in general. For a signal $s$ sufficiently weak compared with the noise $\eta$, the output from the filter can be expressed by the linear approximation as 


$$
\zeta_{k}=F_{k}(\boldsymbol{\eta})+\sum_{l=1}^{N} \frac{\partial F_{k}(\boldsymbol{\eta})}{\partial \eta_{l}} s_{l}
$$

Therefore the signal and noise parts in $\zeta_{k}$ correspond with $S_{k}$ and $\xi_{k}$ in the expression (2.3) as $S_{k}=\left\langle\sum_{l=1}^{N} s_{l} \partial F_{k}(\boldsymbol{\eta}) / \partial \eta_{l}\right\rangle$ and $\xi_{k}=F_{k}(\boldsymbol{\eta})$, respectively.

The input-output characteristics of the optimal nonlinear filter is determined by the functional derivative $\delta R_{X} / \delta F_{k}(\boldsymbol{\eta})=0(k=1, \ldots, N)$ as

$$
F_{k}(\boldsymbol{\eta})=a_{k}-\sum_{l=1}^{N} b_{k l} \frac{\partial \ln \rho(\boldsymbol{\eta})}{\partial \eta_{l}},
$$

where $\boldsymbol{a}=\left(a_{1}, \ldots, a_{N}\right)$ is an arbitrary real vector, $b$ is an arbitrary real regular matrix, and $\rho(\boldsymbol{\eta})$ denotes the joint probability density of the noise $\boldsymbol{\eta}=\left(\eta_{1}, \ldots, \eta_{N}\right)$. The corresponding SNR is given as

$$
R_{X}=\sum_{k, l=1}^{N} I_{k l} s_{k} s_{l}
$$

where $I$ is the Fisher information matrix

$$
I_{k l}=\int \rho(\boldsymbol{\eta}) \frac{\partial \ln \rho(\boldsymbol{\eta})}{\partial \eta_{k}} \frac{\partial \ln \rho(\boldsymbol{\eta})}{\partial \eta_{l}} d \boldsymbol{\eta} .
$$

Note that the resultant SNR is independent of $\boldsymbol{a}$ and $b$. Note also that the optimal filter characteristics is independent of the input signal $s$ in the linear response regime, while the SNR itself depends on the signal $s$. The independence of the optimal nonlinearity from the input signal $s$, which is to be estimated, is the advantage in the practical designing of filters. The optimal filter characteristics is determined only by the knowledge on the noise environment.

The optimal input-output characteristics of the filter Eq. (3.3) straightforwardly shows the possibility of positive use of nonlinear filters in the presence of non-Gaussian noise. If the joint probability density of the noise $\rho(\eta)$ is Gaussian, the optimal input-output characteristics $\boldsymbol{F}(\boldsymbol{\eta})$ becomes linear, and thus any nonlinear filters cannot exceed linear filters in signal processing performance in the presence of Gaussian noise. On the other hand, if $\rho(\eta)$ is non-Gaussian, there exist nonlinear filters that exhibit the SNR higher than linear filters. Therefore, the advantage of nonlinear filter can be obtained only in the presence of non-Gaussian noise.

Since the SNR Eq. (3.4) is given as the quadratic form of the Fisher information matrix $I$, the SNR is evaluated by the decomposition of the input signal $s$ with respect to the eigenvectors of $I$. For white noise, i.e., $\left\langle\eta_{k} \eta_{l}\right\rangle-$ $\left\langle\eta_{k}\right\rangle\left\langle\eta_{l}\right\rangle=\delta_{k l}$, the Fisher information matrix $I$ is diagonal. Furthermore, for stationary white noise, all of the eigenvalues of the matrix $I$ are degenerate. Thus the SNR for sufficiently weak signal is independent of the waveform of the input signal $s$ in the presence of stationary white noise.

\subsection{Optimal nonlinearity for white noise}

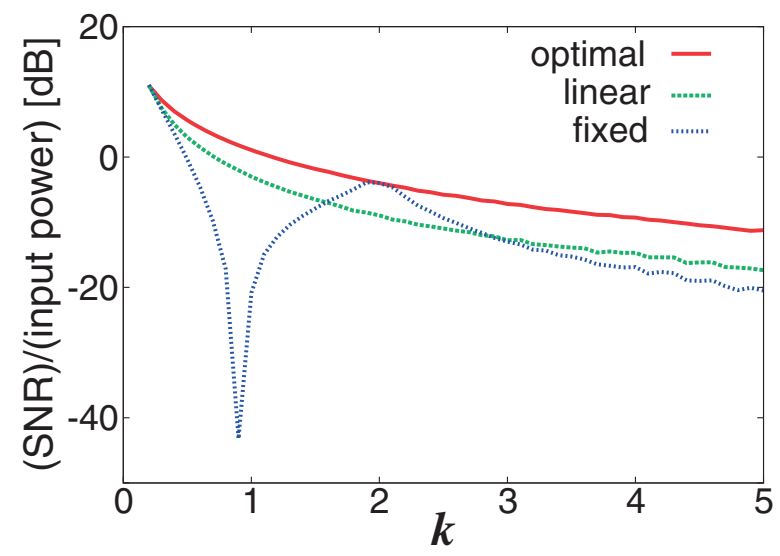

Fig. 2. SNRs obtained by optimal nonlinear filter (red solid line), linear filter (green dotted line), and a nonlinear filter whose inputoutput characteristics is fixed (blue dotted line).

The red solid line in Fig. 2 shows the SNR obtained by the optimal filter in the presence of stationary white mixed Laplace noise as an example of stationary white noise. The joint probability density of the noise is given as

$$
\rho(\boldsymbol{\eta})=\prod_{k=1}^{N} \rho_{1}\left(\eta_{k}\right), \quad \rho_{1}(\eta)=\frac{1}{2}\left(\frac{\exp (-|\eta-k \mu| / k \phi)}{2 k \phi}+\frac{\exp (-|\eta+k \mu| / k \phi)}{2 k \phi}\right),
$$


where $k \mu$ and $k \phi$ characterize the Laplace noise. Note that $k=0$ and $k \rightarrow \infty$ in the probability density Eq. (3.6) mean that the input noise is zero and uniformly random, respectively. In Fig. 2, we fixed the parameters as $\mu=1, \phi=1$, and varies $k$ as a control parameter. As $k$ varies, the probability density of the noise $\rho(\boldsymbol{\eta})$ changes, and the corresponding optimal input-output characteristics $\boldsymbol{F}$ changes. The red solid line in Fig. 2 shows the SNR from such nonlinear filters. For a comparison, the green dotted line in Fig. 2 shows the SNR for a linear filter. As expected, the SNR for the optimal filter is always higher than that of a linear filter. The blue dotted line in Fig. 2 shows the SNR for the nonlinear filter whose input-output characteristics is fixed and optimal for the noise generated from the probability density Eq. (3.6) with $k=2$. In practical devices, it is often the case that the input-output characteristics of the filter is fixed, while the noise environment can change. The blue dotted line in Fig. 2 corresponds with such a situation. Note that the blue dotted line in Fig. 2 exhibits the peak at $k=2$. Such a nonmonotonic change of the SNR with the monotonic change of parameters characterizing noise statistics is called stochastic resonance (SR) [6,7]. According to our results, SR occurs when a filter whose characteristics is fixed is subjected to the noise of which statistics changes. When the given inputoutput characteristics of the device approaches the optimal one, the SNR reaches the local maximum.

\subsection{Optimal nonlinearity for Markovian noise}

In the present subsection, we consider the optimal input-output characteristics for the noise generated from Markovian process. The Markovian noise is practically important and can be found in broad applications, because the physical model of noise is often described by Markovian process. Owing to the simple structure of temporal correlation in Markovian noise, the optimal input-output characteristics of the filter becomes simple and easily implemented.

Consider stationary Markovian process. Let $T\left(\eta_{k+1} \mid \eta_{k}\right)$ be the transition probability of the noise where the value of noise at time $k+1$ becomes $\eta_{k+1}$ when the noise value $\eta_{k}$ is given at time $k$. Then the joint probability density of the temporal sequence of noise $\rho(\boldsymbol{\eta})$ is given as

$$
\rho(\boldsymbol{\eta})=\rho_{\text {ini }}\left(\eta_{1}\right) \prod_{k=1}^{N-1} T\left(\eta_{k+1} \mid \eta_{k}\right),
$$

where $\rho_{\text {ini }}\left(\eta_{1}\right)$ is the probability density of the noise at the initial time $k=1$.

Since the SNR for the optimal filter Eq. (3.4) is independent of the choice of $\boldsymbol{a}$ and $b$, for convenience, we choose these parameters as $\boldsymbol{a}=0$ and $b_{k l}=\delta_{k l}$. Then the optimal input-output characteristics of the filter is given as

$$
F_{k}(\boldsymbol{\eta})=-\frac{\partial \ln T\left(\eta_{k+1} \mid \eta_{k}\right)}{\partial \eta_{k}}-\frac{\partial \ln T\left(\eta_{k} \mid \eta_{k-1}\right)}{\partial \eta_{k}},
$$

for $2 \leq k \leq N-1$,

$$
F_{1}(\boldsymbol{\eta})=-\frac{\partial \ln T\left(\eta_{2} \mid \eta_{1}\right)}{\partial \eta_{1}}-\frac{\partial \ln \rho_{\mathrm{ini}}\left(\eta_{1}\right)}{\partial \eta_{1}}
$$

for $k=1$, and

$$
F_{N}(\eta)=-\frac{\partial \ln T\left(\eta_{N} \mid \eta_{N-1}\right)}{\partial \eta_{N}}
$$

for $k=N$. The SNR obtained from the output of the optimal filter depends on the waveform of the weak input signal $s$. Since the SNR is expressed by the quadratic form of the Fisher information matrix, the upper limit of the achievable SNR is given by the largest eigenvalue of the Fisher information matrix. For Markovian noise, the Fisher information matrix takes the form of tridiagonal matrix. Particularly for stationary Markovian noise, the Fisher information matrix is simply determined by two parameters $A$ and $B$ as

$$
\begin{aligned}
I_{k l} & =A \delta_{k l}+B\left(\delta_{k, l-1}+\delta_{k, l+1}\right), \\
A & =\int \rho(\boldsymbol{\eta})\left(\frac{\partial \ln \rho(\boldsymbol{\eta})}{\partial \eta_{1}}\right)^{2} d^{N} \boldsymbol{\eta}, \\
B & =\int \rho(\boldsymbol{\eta}) \frac{\partial \ln \rho(\boldsymbol{\eta})}{\partial \eta_{1}} \frac{\partial \ln \rho(\boldsymbol{\eta})}{\partial \eta_{2}} d^{N} \boldsymbol{\eta} .
\end{aligned}
$$

The $n$-th eigenvalue for the Fisher information matrix Eq. (3.11), namely $\lambda_{n}$, and its corresponding eigenvector $\boldsymbol{u}^{(n)}$ are given respectively as

$$
\lambda_{n}=A+2 B \cos \frac{n \pi}{N+1}, \quad u_{k}^{(n)}=\sin \frac{n k \pi}{N+1},
$$

where $n=1, \ldots, N$. Thus the upper limit of the achievable SNR, which expresses the maximum signal processing performance of the filter, is $A+2|B|$. The above calculation implies that filtering is efficient to extract a weak signal component $\boldsymbol{u}^{(n)}$ with large $\left|\lambda_{n}\right|$. On the other hand, any filtering techniques are not efficient to extract the signal $\boldsymbol{u}^{(n)}$ with small $\left|\lambda_{n}\right|$. Therefore, in the design of telecommunication systems for example, it is preferred that $\boldsymbol{u}^{(n)}$ with large $\left|\lambda_{n}\right|$ is 
used by a sender of messages. Such preferable signal waveform is determined from the knowledge on the noise environment.

\section{Relation with Estimation Theory}

The input-output characteristics of the optimal nonlinear filter Eq. (3.3) is given by the derivative of the logarithmic likelihood $\partial \ln \rho(\boldsymbol{\eta}) / \partial \eta_{k}(k=1, \ldots, N)$. In addition, the resultant SNR is given by the quadratic form of the Fisher information matrix. These facts imply that the optimal nonlinearity of the filter strongly relates with the signal estimations from the viewpoint of information theory, while the nonlinearity Eq. (3.3) is derived from the viewpoint of the optimization of the SNR, which is originally nothing to do with the information in Shannon's sense.

Consider the estimation of the weak input signal $s$ from the received signal $s+\eta$. Since the output from the optimal filter $\zeta$ is given as Eq. (3.2) in the linear response regime, the natural estimation of the weak signal $s$ is given by the linear estimator as

$$
\hat{s}_{k}=\sum_{l, m=1}^{N} b_{k l}^{-1} I_{l m}^{-1}\left(\zeta_{m}-a_{m}\right)
$$

The error of this linear estimator is evaluated by the covariance matrix $V_{k l}=\left\langle\hat{s}_{k} \hat{s}_{l}\right\rangle-\left\langle\hat{s}_{k}\right\rangle\left\langle\hat{s}_{l}\right\rangle$, which is found to coincide with the inverse of the Fisher information matrix as

$$
V_{k l}=I^{-1} \text {. }
$$

Therefore, the linear estimator Eq. (4.1) exhibits the estimation accuracy coinciding with the Cramér-Rao lower bound. It is concluded that the simple linear estimator Eq. (4.1) of the output from the optimal nonlinear filter, which requires computation much less than Bayesian estimation, gives the high estimation accuracy. This is the advantage in the practical situations such that real time signal processing is required.

\section{Conclusion}

In the present paper, we have derived the optimal input-output characteristics of nonlinear filter that maximizes the SNR obtained from the output. The optimal nonlinearity has been derived for general types of noise at first, and as examples, for stationary white noise and noise generated from Markovian process. The derived nonlinearity implies that there exist nonlinear filters exhibiting the SNR higher than that from linear filters in the presence of non-Gaussian noise, while a linear filter is optimal under Gaussian noise. It is known as the consequence of the fluctuation-dissipation theorem that, in the presence of Gaussian noise, the SNR obtained from nonlinear filters cannot exceed that from linear filters $[8,9]$. On the other hand, it is reported that the SNRs from nonlinear filters exceed that of linear filters in the presence of some types of non-Gaussian noises [10-12]. Our results unify these facts, and elucidate the relation between the signal extraction performance of general types of nonlinear filters and the noise environment. The results will be a guideline in the recent investigations on the positive usage of noise in engineering problems [13-16].

Furthermore, it has been found that the obtained optimal filters yield the simple linear estimator that shows the theoretically highest signal estimation accuracy, i.e., Cramér-Rao lower bound. This result implies that, in the presence of non-Gaussian noise, nonlinear filters can be used for weak signal detection/estimation with high speed signal processing. Therefore, the results give the criterion to design efficient filters in practical engineering problems.

\section{Appendix: Derivation of Eq. (3.3)}

The optimal input-output characteristics Eq. (3.3) is derived by the maximization of the SNR $R_{X}$ defined by Eq. (2.3). The functional derivative of $R_{X}$ with respect to the filter characteristics $F_{i}(\eta)$ is given as

$$
\frac{\delta R_{X}}{\delta F_{i}(\boldsymbol{\eta})}=\sum_{j_{1}, j_{2}, j_{3}, j_{4}=1}^{N}\left(2 \frac{\delta\left\langle\partial_{j_{3}} F_{j_{1}}\right\rangle}{\delta F_{i}(\boldsymbol{\eta})} C_{j_{1} j_{2}}^{-1}\left\langle\partial_{j_{4}} F_{j_{2}}\right\rangle+\left\langle\partial_{j_{3}} F_{j_{1}}\right\rangle \frac{\delta C_{j_{1} j_{2}}^{-1}}{\delta F_{i}(\boldsymbol{\eta})}\left\langle\partial_{j_{4}} F_{j_{2}}\right\rangle\right) s_{j_{3}} s_{j_{4}},
$$

where $\left\langle\partial_{j} F_{l}\right\rangle$ denotes the average of $\partial F_{l}(\eta) / \partial \eta_{j}$. Since the natural boundary condition $\rho(\eta) \rightarrow 0$ is expected to hold in the limit $\eta_{i} \rightarrow \infty$ for arbitrary $i(i=1, \ldots, N)$, the following equations are obtained:

$$
\begin{aligned}
\frac{\delta\left\langle\partial_{l} F_{j}\right\rangle}{\delta F_{i}(\boldsymbol{\eta})} & =-\delta_{i j} \frac{\partial \rho(\boldsymbol{\eta})}{\partial \eta_{l}}, \\
\frac{\delta C_{j l}^{-1}}{\delta F_{i}(\boldsymbol{\eta})} & =-\sum_{m_{1}, m_{2}=1}^{N} C_{j m_{1}}^{-1}\left[\delta_{m_{1} i}\left(F_{m_{2}}(\boldsymbol{\eta})-\left\langle F_{m_{2}}\right\rangle\right)+\delta_{i m_{2}}\left(F_{m_{1}}(\boldsymbol{\eta})-\left\langle F_{m_{1}}\right\rangle\right)\right] \rho(\boldsymbol{\eta}) C_{m_{2} l}^{-1},
\end{aligned}
$$

where the property $\langle\cdots\rangle=\int \rho(\eta) \cdots d \eta$ has been used. Using these equations, the maximum condition $\delta R_{X} / \delta F_{i}(\eta)=0$ becomes 


$$
\frac{\partial \ln \rho(\boldsymbol{\eta})}{\partial \eta_{i}}-\sum_{j_{1}, j_{2}=1}^{N}\left(F_{j_{1}}(\boldsymbol{\eta})-\left\langle F_{j_{1}}\right\rangle\right) C_{j_{1} j_{2}}^{-1}\left\langle\partial_{i} F_{j_{2}}\right\rangle=0 .
$$

This result implies that $F_{i}(\boldsymbol{\eta})-\left\langle F_{i}\right\rangle$ is given by a linear combination of $\partial \ln \rho(\boldsymbol{\eta}) / \partial \eta_{l}$. Then Eq. (3.3) is derived with arbitrary constants $\boldsymbol{a}$ and $b$, which are self-consistently determined by the moments of $F_{i}(\boldsymbol{\eta})$.

\section{REFERENCES}

[1] Oppenheim, A. V., Schafer, R. W., and Buck, J. R., Discrete-Time Signal Processing, Prentice Hall International (1989).

[2] Kalman, R. E., “A new approach to linear filtering and prediction problems," J. Basic Eng., 82: 35-45 (1960).

[3] Ichiki, A., and Tadokoro, Y., "Relation between optimal nonlinearity and non-Gaussian noise: Enhancing a weak signal in a nonlinear system," Phys. Rev. E, 87: 012124 (2013).

[4] Rao, C. R., "Information and the accuracy attainable in the estimation of statistical parameters," Bull. Calcutta Math. Soc., 37: 81-91 (1945)

[5] Cramér, H., Mathematical Methods of Statistics, Princeton University Press (1946).

[6] Benzi, R., Sutera, A., and Vulpiani, A., "The mechanism of stochastic resonance," J. Phys. A: Math. Gen., 14: L453-L457 (1981).

[7] Gammaitoni, L., Hänggi, P., Jung, P., and Marchesoni, F., "Stochastic resonance," Rev. Mod. Phys., 70: $223-287$ (1998).

[8] Dykman, M. I., Luchinsky, D. G., Mannella, R., McClintock, P. V. E., Stein, N. G., and Stocks, N. G., "Stochastic resonance in perspective," Nuovo Cim. D, 17: 661-683 (1995).

[9] DeWeese, M., and Bialek, W., "Information flow in sensory neurons," Nuovo Cim. D, 17: 733-741 (1995).

[10] Gammaitoni, L., "Stochastic resonance and the dithering effect in threshold physical systems," Phys. Rev. E, 52: 4691-4698 (1995).

[11] Chapeau-Blondeau, F., and Godivier, X., "Theory of stochastic resonance in signal transmission by static nonlinear systems," Phys. Rev. E, 55: 1478-1495 (1997).

[12] Kosko, B., and Mitaim, S., "Robust stochastic resonance: Signal detection and adaptation in impulsive noise," Phys. Rev. E, 64: 051110 (2001).

[13] Mitaim, S., and Kosko, B., “Adaptive stochastic resonance," Proc. IEEE, 86: 2152-2183 (1998).

[14] Chapeau-Blondeau, F., and Rousseau, D., "Noise-enhanced performance for an optimal Bayesian estimator," IEEE Trans. Signal Process., 52: 1327-1334 (2004).

[15] Chen, H., Varshney, P. K., Kay, S. M., and Michels, J. H., "Theory of the stochastic resonance effect in signal detection: Part I-Fixed detectors," IEEE Trans. Signal Process., 55: 3172-3184 (2007).

[16] Sugiura, S., Ichiki, A., and Tadokoro, Y., "Stochastic-resonance based iterative detection for serially concatenated turbo codes," IEEE Signal Process. Lett., 19: 655-658 (2012). 